\title{
Repercusión de la pandemia COVID-19 en la práctica de la cardiología intervencional en Chile. Datos aportados por la encuesta del grupo Stent-Save a Life! LATAM working group.
}

\author{
Christian Backhouse ${ }^{1}$, Jorge Sandoval${ }^{2}$, Gonzalo Martínez ${ }^{3}$, Gabriel Maluenda $^{4}$, Nicolás Veas ${ }^{5}$, Nilo Carvajal $^{6}$, Juan Ortega ${ }^{7}$, Alejandro Fleming $^{8}$, \\ Marcio Alvarado ${ }^{9}$, Aníbal Domínguez ${ }^{10}$, Christian Pincetti ${ }^{11}$, Carlos Olivares ${ }^{11}$, César Vargas ${ }^{12}$, Víctor Assef ${ }^{13}$, Ángel Puentes ${ }^{1}$. \\ 1. Unidad de Hemodinamia, Centro de Enfermedades Cardiovasculares, Hospital San Juan de Dios, Santiago. \\ 2. Instituto Nacional del Tórax, Santiago. \\ 3. División de Enfermedades Cardiovasculares, Pontificia Universidad Católica de Chile, Santiago. \\ 4. Hospital San Borja Arriarán - Universidad de Chile, Santiago. \\ 5. Unidad de Cardiología Intervencional y Hemodinamia, Hospital Sótero del Río, Santiago. \\ 6. Unidad de Hemodinamia, Clínica Indisa, Santiago. \\ 7. Unidad de Hemodinamia, Hospital Dr. Juan Noé Crevani, Arica. \\ 8. Unidad de Cardiología, Hospital de La Serena, La Serena. \\ 9. Unidad de Cardiología Intervencional, Hospital Libertador Bernardo O'higgins, Rancagua. \\ 10. Unidad de Hemodinamia, Hospital Dr. Víctor Ríos Ruiz, Los Ángeles. \\ 11. Unidad de Hemodinamia, Hospital Hernán Henríquez Aravena, Temuco. \\ 12. Unidad de Hemodinamia, Clínica Alemana - Hospital Base San José, Osorno. \\ 13. Unidad de Hemodinamia, Hospital Puerto Montt, Puerto Montt. \\ Financiamiento: No \\ Conflictos de Interés: No
}

Introducción: El Stent-Save a Life! (SSL) LA-

TAM working group diseñó una encuesta para objetivar la reducción de la actividad de los laboratorios de hemodinamia en Latinoamérica durante la pandemia COVID-19. Ante la amenaza de nuevos confinamientos en Chile, nos propusimos objetivar las consecuencias de la primera ola de contagios en nuestra actividad.

Objetivos: Discutir la repercusión de la pandemia en la cardiología intervencional en Chile.

Métodos: El grupo SSL realizó una encuesta telemática a todos los países de Latinoamérica incluido Chile. Se registraron las coronariografías (CAG), intervenciones coronarias percutáneas (ACTP) e intervenciones estructurales, comparando dos períodos determinados por el confinamiento por la pandemia, cada uno de dos semanas. Pre-COVID-19: período previo al confinamiento, y COVID-19: período durante el confinamiento. Se analizan, a partir de esta en- cuesta, los resultados aplicados a nuestro país.

Resultados: Se obtuvo respuesta de trece centros. Hubo una reducción en el número global de procedimientos entre período Pre-COVID-19 y COVID-19 de un $65,1 \%$. Se reportó una disminución de $67 \%$ en las CAG, de un 59,4\% en las ACTP y de un $92 \%$ en los procedimientos terapéuticos estructurales. Entre ambos períodos se redujo la consulta por Síndrome Coronario Agudo por elevación del segmento ST (SCACEST) en $40,8 \%$.

Conclusiones: En nuestro país se objetivó una reducción marcada de la actividad asistencial de la cardiología intervencional durante la pandemia COVID-19 y una disminución significativa en el número de pacientes tratados por SCACEST. Los resultados de nuestro país son similares a los reportados por países de Latinoamérica, Europa y Norteamérica.

Palabras Clave: Pandemia COVID-19; confinamiento; cardiología intervencional. 


\section{Decrease in the activity of interventional cardiology in Chile: a report from the "Stent-Save a Life! LATAM working group"}

Background: The Stent-Save a Life! (SSL) LATAM working group designed a survey to demonstrate the reduction in the activity of cardiac catheterization laboratories in Latin America during the COVID-19 pandemic. Considering the risk of a new confinement in Chile, we decided to assess the impact of the first wave of contagions on our activity.

Aims: To discuss the repercussion of the COVID-19 pandemic on the activity of interventional cardiology in Chile.

Methods: The SSL group conducted a telematic survey in all Latin American countries. Coronary angiography, coronary interventions (PCI) and structural interventions were registered, comparing two periods of two weeks duration each: before and during COVID-19 confinement. Results obtained in Chile are analyzed.
Results: Thirteen centers in Chile answered the survey. There was an overall decrease of $65.1 \%$ in the number of procedures between the pre and the post COVID-19 periods. Coronary angiographies decreased $67 \%$, PCI $59.4 \%$ and therapeutical structural procedures $92 \%$. The reduction in acute coronary syndrome with ST segment elevation (STEMI) was $40,8 \%$ between periods.

Conclusions: In Chile, a significant reduction in healthcare activity related to interventional cardio$\operatorname{logy}$ and a significant decrease in the number of patients treated with STEMI was observed during the COVID-19 pandemic. The results are similar to those reported by Latin American, European and North American countries.

Key Words: COVID-19 pandemic; confinement; interventional cardiology. 


\section{Introducción:}

La pandemia COVID-19 por el virus SARS-CoV-2 ha generado una saturación del sistema de salud, especialmente a nivel de las unidades de emergencia y cuidados intensivos ${ }^{1}$. Esta realidad evidenciada inicialmente en Asia y Europa, ha impactado significativamente en Latinoamérica, obligando a sus países a reestructurar las medidas sanitarias de forma continua. Lo anterior hace plantear que pacientes con patología cardiovascular podrían verse afectados, sin recibir la atención adecuada tanto en prestaciones ambulatorias electivas como en los procedimientos de urgencia con necesidad de hospitalización. Es en este grupo donde los Síndromes Coronarios Agudos (SCA) y particularmente aquellos que cursan con elevación del segmento ST (SCACEST) se benefician de intervenciones coronarias percutáneas ${ }^{2}$.

Ante el aumento exponencial de casos nuevos en el país y las amenazas de nuevos confinamientos, es relevante cuantificar lo ocurrido en Chile durante el primer período de contagios del año 2020. Esta nueva realidad ha sido objetivada en países de Europa y Norteamérica, regis- trándose una menor cantidad de procedimientos intervencionales realizados durante la pandemia. En España, Rodríguez-Leor y colaboradores, publicaron recientemente una disminución significativa en el número de procedimientos diagnósticos de un $56 \%{ }^{3}$. En Estados Unidos, García y colaboradores, reportaron un disminución de un $38 \%$ en la utilización de laboratorios de hemodinamia por SCACEST ${ }^{4}$. Además, se ha reportado que el temor que le genera a los pacientes consultar en los hospitales, ha desencadenado un retardo en la consulta, como lo reportó Tam y colaboradores en un registro Chino ${ }^{5}$. La iniciativa Stent Save a Life! (SSL) del LATAM Working Group en acuerdo con SOLACI ha realizado una encuesta en diversos países de América Latina con la intención de reportar la repercusión de la pandemia COVID-19 en la región ${ }^{6}$. En el mencionado reporte aportaron información países como Argentina, Uruguay y Brasil, entre otros. Chile apoyó con trece centros.

Lo que expondremos a continuación, en cooperación con el programa SSL, es el análisis de la encuesta realizada por este grupo aplicado a nuestro país.

Figura 1. Formulario de la Información solicitada en la encuesta internacional en América Latina sobre la práctica de la cardiología intervencionista durante la pandemia COVID-19

\begin{tabular}{|c|c|c|c|c|c|c|c|c|}
\hline \multicolumn{9}{|c|}{ Cambios en la actividad del centro. Registrar el número de casos en cada periodo. } \\
\hline Periodos & CAG (n) & CAG por SCA (n) & \multicolumn{2}{|c|}{$\operatorname{ACTP}(n)$} & ACTP en SCACEST ( $n)$ & \multicolumn{3}{|c|}{ Terapia Estructural: VLP, TAVI, cierre CIA,FOP, etc. (n) } \\
\hline \multicolumn{9}{|c|}{$\begin{array}{l}\text { Pre-COVID-19 } \\
\text { COVID }\end{array}$} \\
\hline \multicolumn{9}{|c|}{ Cambios observados en el manejo del Infarto de Miocardio con elevación del ST durante el periodo COVID-19. } \\
\hline \multicolumn{3}{|c|}{ Percepción respecto a la reperfusión } & \multicolumn{2}{|c|}{ Es igual } & Es menor & Es mayor & \multicolumn{2}{|c|}{ No tengo respuesta } \\
\hline \multicolumn{9}{|c|}{ El uso de trombolíticos en el SCACEST: } \\
\hline \multicolumn{9}{|c|}{ El número de SCACEST diagnosticados: } \\
\hline \multicolumn{9}{|c|}{ La demora a la reperfusión: } \\
\hline \multicolumn{9}{|c|}{ Cambios en la internación post-procedimiento durante el periodo COVID-19. } \\
\hline & & \multicolumn{2}{|c|}{ No hay cambios } & \multicolumn{2}{|c|}{$\begin{array}{l}\text { Se trata de derivarlos a otros } \\
\text { hospitales por protocolo }\end{array}$} & \multicolumn{2}{|c|}{$\begin{array}{l}\text { Se derivan a otros hospitales } \\
\text { por falta de camas }\end{array}$} & No tengo respuesta \\
\hline \multicolumn{2}{|c|}{$\begin{array}{l}\text { Respecto a la internación } \\
\text { post-procedimiento: }\end{array}$} & & & & & & & \\
\hline
\end{tabular}

Personal afectado por COVID-19. Referir la presencia de afectados en el Centro de Hemodinamia

$\begin{aligned} & \text { Respecto al COVID-19, } \\ & \text { ¿cuál es la situación en su servicio?: }\end{aligned}$
$\begin{aligned} & \text { ¿Hay integrantes del equipo de baja por } \\ & \text { contagio o contacto laboral cercano? }\end{aligned}$

CAG: coronariografia; SCA: Síndrome Coronario Agudo; SCACEST: síndrome coronario agudo con elevación del segmento ST; ACTP: angioplastia coronaria transluminal percutánea; VLP: valvuloplastia; TAVI: implante percutáneo de válvula aórtica; CIA: comunicación interauricular; FOP: foramen oval permeable. 


\section{Métodos:}

El grupo SSL solicitó a través de una encuesta de opinión evaluar la actividad en los centros de hemodinamia de América Latina en una quincena previa y en otra posterior a la declaración del confinamiento social por la pandemia de COVID-19 (Figura 1). En el caso de Chile se consideró como quincena Pre-COVID-19 entre el lunes 24 de Febrero y el domingo 8 de Marzo del 2020, y como quincena COVID-19 entre el lunes 30 de Marzo y el domingo 12 de Abril de 2020. La encuesta se envió el 17 de abril, recibiéndose respuesta hasta el 30 de abril de 2020. Ésta consideraba tanto datos objetivos sobre variación en el número de procedimientos, como la percepción subjetiva de los profesionales de salud sobre variables donde estaba repercutiendo la pandemia en el manejo de los pacientes.

Los centros de Chile que aportaron con dicha información fueron:

- Hospital Juan Noé Crevani de Arica

- Hospital de La Serena

- Instituto Nacional del Tórax de Santiago

- Hospital San Juan de Dios de Santiago

- Hospital San Borja Arriarán de Santiago

- Pontificia Universidad Católica de Santiago

- Hospital Sótero del Río de Santiago

- Clínica Indisa de Santiago

- Hospital Libertador Bernardo O’higgins de Rancagua

- Hospital Víctor Ríos Ruiz de Los Ángeles
- Hospital Hernán Henríquez Aravena de Temuco

- Hospital Base San José de Osorno

- Hospital de Puerto Montt

La información se presentó como un estudio descriptivo observacional. Para el análisis de los datos se utilizó la herramienta Google Forms, autoajustable a multi-plataformas. Se reportan los datos objetivos aportados por la encuesta referente a nuestro país.

\section{Resultados:}

En Chile, la situación registrada por los centros entrevistados documentó en la fase Pre-COVID un total de 1354 procedimientos y en fase COVID un total de 473 , lo que representa una disminución de un $65,1 \%$.

\section{A continuación, se presenta el desglose según el tipo de procedimiento:}

\section{Coronariografías:}

El número de coronariografías (CAG), disminuyó de 895 en época Pre-COVID a 295 en época COVID, lo que traduce una reducción de $67 \%$. Si consideramos los procedimientos diagnósticos electivos, en fase Pre-COVID se realizaron 495 y en fase COVID sólo 76 procedimientos, lo que significa una caída de $84,6 \%$. En el contexto del SCA, las CAG realizadas en período Pre-COVID fueron 400 y en período COVID sólo 219, una reducción de $45,3 \%$.

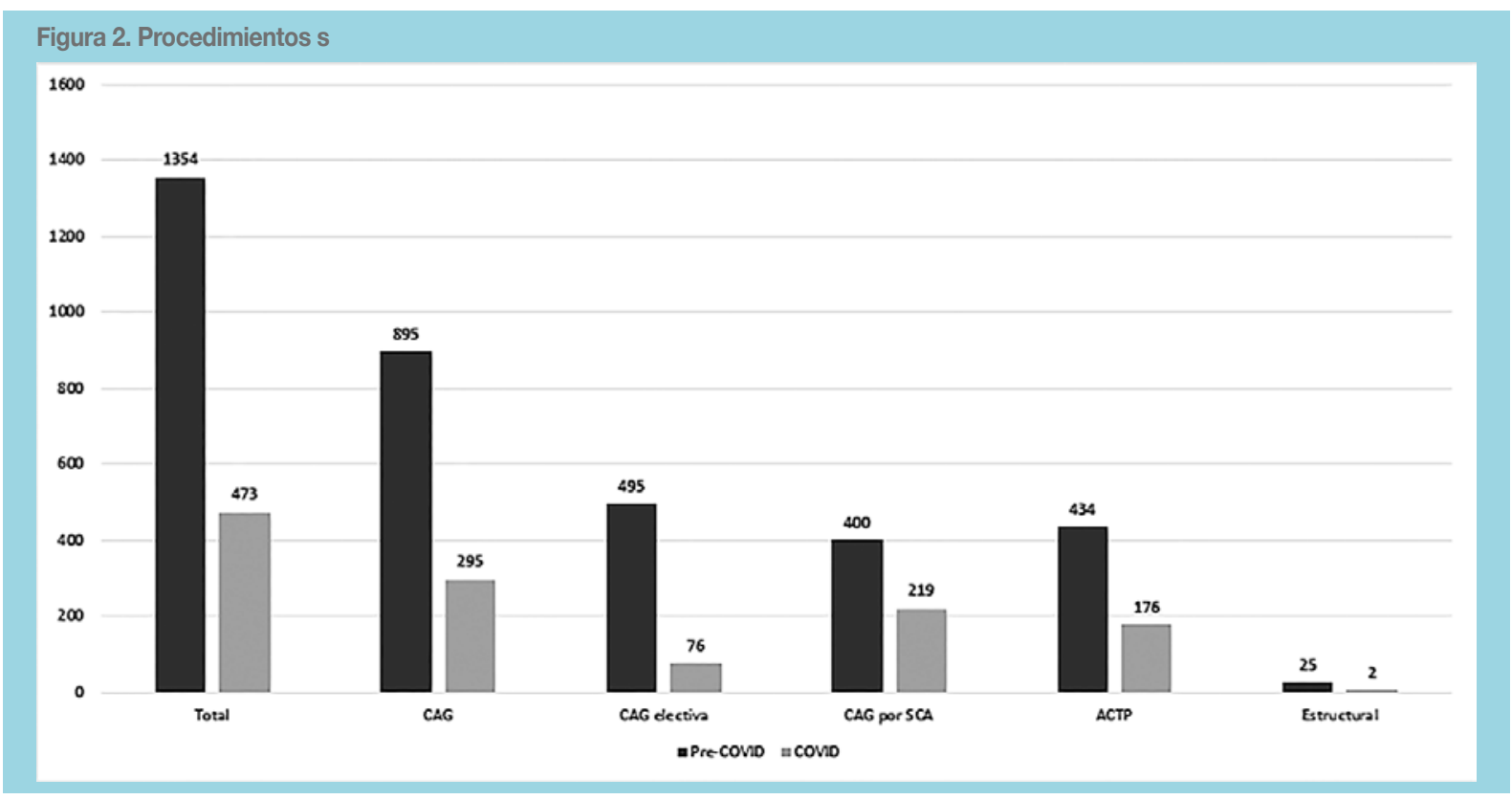




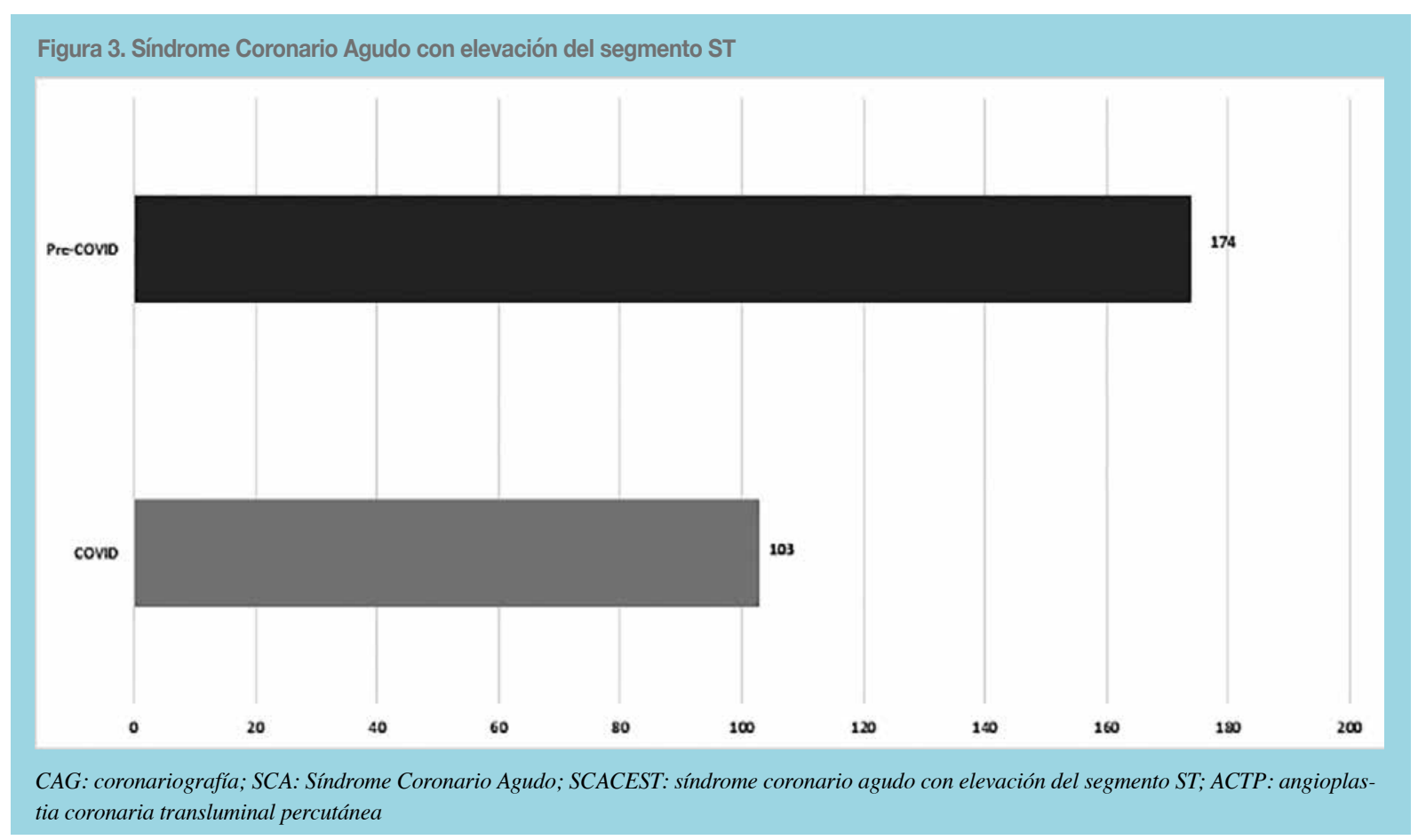

\section{Angioplastía Coronaria Percutánea:}

El número total de Angioplastías Coronarias Transluminales Percutáneas (ACTP) disminuyó de 434 en fase Pre-COVID a 176 en fase COVID, lo que representa una reducción de 59,4\%. El SCACEST se redujo de 174 a 103 procedimientos en el mismo período, lo que representa una disminución de 40,8\%.

\section{Procedimientos Estructurales:}

Se consideró en este grupo la valvuloplastía mitral y aórtica, cierre de comunicación interatrial, cierre de foramen oval permeable e implante percutáneo de válvula aórtica. De lo anterior, se objetiva una reducción de 25 procedimientos estructurales realizados en época Pre-COVID a sólo 2 procedimientos en época COVID, lo que traduce una disminución de un $92 \%$. (Figuras 2 y 3 )

\section{Discusión:}

Los resultados reportados muestran una disminución significativa de todos los procedimientos realizados en las salas de hemodinamia en nuestro país, lo que concuerda con lo reportado por el grupo SSL para Latinoamérica y con lo reportado por otros países que enfrentaron previamente esta pandemia.

Con respecto a estudios internacionales, el más completo es el reportado por el grupo español liderado por Rodrí-
guez-Leor y colaboradores ${ }^{3}$, que incluyeron 17 comunidades y 81 centros a lo largo del país. Ellos reportaron un $57 \%$ de reducción de procedimientos diagnósticos electivos, un $48 \%$ de disminución de los terapéuticos coronarios y una reducción de un $81 \%$ de procedimientos estructurales. Si comparamos lo anterior con nuestra realidad, evidenciaremos que los números presentados en Chile son levemente mayores en cada uno de los ítems. Con respecto al SCACEST el grupo español documentó un $40 \%$ de disminución, similar a lo reportado por nuestro país. En Estados Unidos el grupo liderado por García y colaboradores ${ }^{4}$, recabó información de 9 centros del país con un volumen significativo de procedimientos y reportó una reducción de 38\%, similar a lo ya documentado. En cuanto a la información reportada por el grupo $\mathrm{SSL}^{6}$, las CAG disminuyeron en un $65,2 \%$, las CAG por SCA en $55,7 \%$ y las ACTP en un 59,4\%, muy similar a lo reportado por Chile. En cuanto a la presentación por SCACEST existe una reducción de un $51,2 \%$, mayor a lo reportado por nuestro país.

Esta disminución en la actividad de los laboratorios era algo previsible y confirma la percepción subjetiva de los profesionales que trabajan en el área y que fue corroborado por los centro que participaron en esta encuesta. El llamado de grupos internacionales a suspender procedimientos electivos durante la pandemia y a manejar 
médicamente los SCA sin elevación del segmento ST (SCASEST) de bajo riesgo podrían apoyar los datos reportados. Lo que es llamativo, y que no guarda relación con lo anterior, es la reducción promedio de un $40 \%$ de los SCACEST en período de confinamiento por la pandemia. Una probable explicación a esto es el temor presentado por los pacientes que cursan con dicha patología al ser hospitalizados en centros de salud colapsados. Lo anterior podría resultar en un aumento de casos de SCACEST de reperfusión tardía o de manejo médico. Esto fue reportado recientemente en un registro Chino de la ciudad de Hong Kong, donde Tam y colaboradores ${ }^{5}$, demostraron un retardo en la consulta promedio desde el inicio de la sintomatología de 82,5 minutos a $318 \mathrm{minu}$ tos en horario de consulta diurna. El grupo $\mathrm{SSL}^{6}$ hace hincapié en que el estudio no aclara sobre cuáles fueron los motivos de la disminución del SCACEST. Lo que sí es evidente es que esto conllevará un aumento de la mor- talidad cardiovascular, insuficiencia cardiaca, arritmias y nuevos eventos cardiovasculares. Será necesario tomar conciencia sobre la situación de estos pacientes durante la evolución de la pandemia, sobre todo ante el aumento de casos nuevos y amenazas de confinamientos que ocurren en Chile en la actualidad.

Finalmente, es importante resaltar que la presente encuesta fue voluntaria para todos los centros médicos del país y que la información presentada es sólo de los laboratorios que contestaron la encuesta.

\section{Conclusiones:}

Los datos reportados en la encuesta del grupo SSL demuestran que en Chile la actividad de los laboratorios de hemodinamia se ha visto reducida significativamente, al igual que el número de angioplastías primarias por SCACEST durante el período de confinamiento en la pandemia COVID-19.

\section{Referencias:}

1. CENTERS FOR DISEASE CONTROL AND PREVENTION CORONAVIRUS DISEASE 2019 (COVID-19). Available at:. https://www.cdc.gov/coronavirus/2019-ncov/hcp/guidance-hcf.html?CDC_AA_refVal=https $\% 3 \mathrm{~A} \% 2 \mathrm{~F} \% 2 \mathrm{Fwww} . c d c$. gov $\% 2$ Fcoronavirus $\% 2$ F2019-ncov $\% 2$ Fhealthcare-facilities\%2Fguidance-hcf.html.

2. IBANEZ B, JAMES S, AGEWALL S, et al. 2017 ESC Guidelines for the Management of Acute Myocardial Infarction in Patients Presenting With ST-Segment Elevation: The Task Force for the Management of Acute Myocardial Infarction in Patients Presenting With ST-Segment Elevation of the European Society of Cardiology (ESC). Eur Heart J; Aug 26, 2017. https://doi.org/10.1093/eurheartj/ehx393.

3. RODRÍGUEZ-LEOR O et al. Impacto de la pandemia de COVID-19 sobre la actividad asistencial en cardiología intervencionista en España. REC Interv Cardiol. 2020;2(2):82-89. https://doi.org/10.24875/RECIC.M20000120.
4. GARCIA S et al. Reduction in ST-Segment Elevation Cardiac Catheterization Laboratory Activations in the United States during COVID-19 Pandemic. J Am Coll Cardiol. 2020 Jun, 75 (22) 2871-2872. https://doi.org/10.1016/j. jacc.2020.04.011.

5. TAM CF et al. Impact of coronarvirus disease 2019 (COVID-19) outbreak on ST-segment elevation myocardial infarction care in Hong-Kong, China. Cardiovasc Qual Outcomes. March, 2020. https://doi.org/10.1161/CIRCOUTCOMES.120.006631.

6. ARTUCIO C et al. Una encuesta internacional en América Latina sobre la práctica de la cardiología intervencionista durante la pandemia COVID-19, con especial enfoque en el infarto de miocardio y el estado sanitario del equipo de salud. Stent-Save a Life! LATAM working group, en acuerdo con la SOLACI (Sociedad Latinoamericana de Cardiología Intervencionista). 